\title{
Distribution of Data Dissemination Model using DTN Routing Protocols
}

\author{
S. Parameswari, K. Kavitha, P. Elango
}

\begin{abstract}
In this research paper compare the protocol's performance together with the experimental results of optimal routing using real-life scenarios of vehicles and pedestrians roaming in a city. In this research paper, conduct several simulation comparison experiments(in the NS2 Software) to show the impact of changing buffer capacity, packet lifetime, packet generation rate, and number of nodes on the performance metrics. This research paper is concluded by providing guidelines to develop an efficient DTN routing protocol. To the best of researcher(Parameswari et al.,) knowledge, this work is the first to provide a detailed performance comparison among the diverse collection of DTN routing protocols.
\end{abstract}

Keywords : DTN, Fog Route, IoT, Delay Tolerant.

\section{INTRODUCTION}

Fog computing is a medium weight and intermediate level of computing power. Rather than a substitute, fog computing often serves as a complement to cloud computing. Fog computing concept, actually a cloud computing close to the 'ground', creates automated response that drives the value. Both cloud and fog provide data, computation, storage and application services to end-users.

However, fog can be distinguished from cloud by its proximity to end-users, the dense geographical distribution and its support for mobility. Fog computing typically has a three-tier mobile-fog-cloud structure (Luan et al., 2015). In the mobile tier, it could include all wireless devices such as smartphones, tablets, laptops. In the fog tier, fog servers provide services to the end users and synchronize data with the cloud. In the cloud tier, cloud provider provides content service required by geodistributed fog servers. Data dissemination between a mobile user and a fog server is occurred when this mobile user retrieves content. If this fog server has the required content, it sends the content to the mobile user. Otherwise, this fog server needs to send a query to its cloud provider to find and download it into its local storage. On another side, fog servers need to regularly check

Revised Manuscript Received on February 15, 2020.

* Correspondence Author

S. Parameswari*, Research Scholar, Dept. of Computer Science and Engineering, Annamalai University, Annamalai Nagar 608 002.India.

Dr.K. Kavitha, Research Superviosr/Assoc. Professor, Dept. of Computer Science and Engineering, Annamalai University, Annamalai Nagar 608 002. India.

Dr.P. Elango, Asst. Professor, Perunthalaivar Kamarajar Institute of Engineering \& Technology (PKEIT), Karaikal, India.

(c) The Authors. Published by Blue Eyes Intelligence Engineering and Sciences Publication (BEIESP). This is an open access article under the CC BY-NC-ND license (http://creativecommons.org/licenses/by-nc-nd/4.0/) with their cloud providers whether the fog servers have the updated contents or not; if not, they need to update their storage by retrieving from the cloud via. either wired or wireless networks, e.g., cellular networks. Such data disseminations may involve a huge cost due to the large data volume.

\section{A. Analysis of the Algorithm}

In this section, here it will analyze the convergence and optimality of the LAB scheme in the feasible set of Problem P1.

Lemma 1. When $\tilde{\eta}^{K+1} \neq \tilde{\eta}^{k}, \tilde{\eta}^{k+1}$ provides a descent direction for $L(\tilde{\eta})$ at $\tilde{\eta}^{k}$.

$$
\text { Proof: As } 0 \leq \tilde{\eta}_{j}^{k}(x) \leq 1, L(\tilde{\eta}) \text { is defined in } \widehat{F}
$$
As shown in Lemma $1, L(\tilde{\eta})$ is a convex function of $\tilde{\eta}$, and thus we need to prove $\left(\nabla L\left(\tilde{\eta}^{k}\right), \tilde{\eta}^{k+1}-\tilde{\eta}^{k}\right)<0$ : Thus, we have

$$
\begin{aligned}
& \left\langle\nabla L\left(\tilde{\eta}^{k}\right), \tilde{\eta}^{k+1}-\tilde{\eta}^{k}\right\rangle \\
& =\int_{x \in \mathscr{A}} \sum_{\mathrm{j} \in \mathcal{J}} \lambda(\mathrm{x}) \mathrm{v}(\mathrm{x}) \frac{\tilde{\mathrm{\eta}}_{\mathrm{j}}^{\mathrm{k}+1}(\mathrm{x})-\tilde{\mathrm{\eta}}_{\mathrm{j}}^{\mathrm{k}}(\mathrm{x})}{\mathrm{C}_{\mathrm{j}} \mathrm{r}_{\mathrm{j}}(\mathrm{x}) \phi_{\mathrm{j}}(\mathrm{k})} \\
& =\int_{x \in \mathscr{A} l} \lambda(\mathrm{x}) \mathrm{v}(\mathrm{x}) \sum_{\mathrm{j} \in \mathcal{J}} \frac{\tilde{\eta}_{\mathrm{j}}^{\mathrm{k}+1}(\mathrm{x})-\tilde{\mathrm{\eta}}_{\mathrm{j}}^{\mathrm{k}}(\mathrm{x})}{\mathrm{C}_{\mathrm{j}} \mathrm{r}_{\mathrm{j}}(\mathrm{x}) \phi_{\mathrm{j}}(\mathrm{k})} \\
& \text { Based on Eq. (1), here } \\
& \tilde{\eta}_{\mathrm{j}}^{\mathrm{k}+1}(\mathrm{x})-\tilde{\eta}_{\mathrm{j}}^{\mathrm{k}}(\mathrm{x})=(1-\beta)\left(\eta_{\mathrm{j}}^{\mathrm{k}}(\mathrm{x})-\tilde{\eta}_{\mathrm{j}}^{\mathrm{k}}(\mathrm{x})\right)
\end{aligned}
$$

As we know,

$$
\eta_{j}^{k}(x)=\left\{\begin{array}{l}
1, \text { ifj }=p^{k}(x) \\
0, \text { ifj } \neq p^{k}(x) .
\end{array}\right.
$$

Owing to the BS selection rule at the user side in the kth iteration, i.e., $\mathrm{p}^{\mathrm{k}}(\mathrm{x})=\arg \max _{\mathrm{j} \in \mathcal{J}} \mathrm{C}_{\mathrm{j}} \mathrm{r}_{\mathrm{j}}(\mathrm{x}) \phi_{\mathrm{j}}(\mathrm{k})$,we can derive

$$
\begin{aligned}
& \sum_{j \in j}(1-\beta) \frac{\eta_{j}^{k}(x)-\tilde{\eta}_{j}^{k}(x)}{C_{j} r_{j}(x) \phi_{j}(k)} \leq 0 \\
& \text { Since } \tilde{\eta}^{K+1} \neq \tilde{\eta}^{k},
\end{aligned}
$$


$\sum_{j \in j}(1-\beta) \frac{\eta_{j}^{k}(x)-\eta_{j}^{k}(x)}{C_{j} r_{j}(x) \phi_{j}(k)}<0$

Hence, we have proved $\left\langle\nabla L\left(\tilde{\eta}^{\mathrm{k}}\right), \tilde{\eta}^{\mathrm{k}+1}-\tilde{\eta}^{\mathrm{k}}\right\rangle<0$

Meanwhile, as the LAB scheme is executed iteratively, we will also analyze if the BS selection rule at the IoT device side in each iteration is the best option by proving the following theorem.

Theorem 1. Given the advertised traffic loads of BSs and computing loads of fog nodes, the optimal IoT device association rule at the IoT device side is:

$$
\mathrm{p}^{\mathrm{k}}(\mathrm{x})_{=a r g} \max _{\mathrm{jeJ}} \mathrm{C}_{\mathrm{j}} \mathrm{r}_{\mathrm{j}}(\mathrm{x}) \phi_{\mathrm{j}}(\mathrm{k}) \text {, }
$$

Proof: In the kth iteration, $\eta^{\mathbf{k}}$ is the IoT device association achieved by the proposed IoT device side algorithm: $\mathrm{p}^{\mathrm{k}}(\mathrm{x})=\arg \max _{\mathrm{j} \in \mathcal{J}} \mathrm{C}_{\mathrm{j}} \mathrm{r}_{\mathrm{j}}(\mathrm{x}) \phi_{\mathrm{j}}(\mathrm{k})$. Meanwhile,

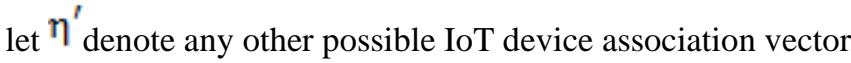
in the iteration. Thus, to prove this theorem, we just need to prove that cannot reduce $\mathrm{L}(\eta)$ any more as compared to $\eta^{\mathrm{k}}$,i.e., $\left\langle\nabla L\left(\eta^{\mathrm{k}}\right), \eta^{\prime}-\eta^{\mathrm{k}}\right\rangle \geq 0$

$$
\left(\nabla \mathrm{L}\left(\eta^{\mathrm{k}}\right), \eta^{\prime}-\eta^{\mathrm{k}}\right)
$$

$$
=\int_{x \in \mathscr{A}} \sum_{\mathrm{j} \in \mathcal{J}} \lambda(\mathrm{x}) \mathrm{v}(\mathrm{x})\left(\eta_{\mathrm{j}}^{\prime}(\mathrm{x})-\eta_{\mathrm{j}}^{\mathrm{k}}(\mathrm{x})\right) \frac{1}{\mathrm{C}_{\mathrm{j}} \mathrm{r}_{\mathrm{j}}(\mathrm{x}) \phi_{\mathrm{j}}(\mathrm{k})} \mathrm{dx}
$$$$
=\int_{\mathrm{x} \in \mathscr{A} \mathcal{A}} \lambda(\mathrm{x}) \mathrm{v}(\mathrm{x}) \sum_{\mathrm{j} \in \mathcal{J}}\left(\eta_{\mathrm{j}}^{\prime}(\mathrm{x})-\eta_{\mathrm{j}}^{\mathrm{k}}(\mathrm{x})\right) \frac{1}{\mathrm{C}_{\mathrm{j}} \mathrm{r}_{\mathrm{j}}(\mathrm{x}) \phi_{\mathrm{j}}(\mathrm{k})} \mathrm{dx} .
$$

Since

$$
\begin{aligned}
\mathrm{p}^{\mathrm{k}}(\mathrm{x})_{=\arg } \max _{\mathrm{j} \in \mathcal{J}} \mathrm{C}_{\mathrm{j}} \mathrm{r}_{\mathrm{j}}(\mathrm{x}) \phi_{\mathrm{j}}(\mathrm{k}), \\
\eta_{\mathrm{j}}^{\mathrm{k}}(\mathrm{x})=\left\{\begin{array}{l}
1, \text { ifj }=\mathrm{p}^{\mathrm{k}}(\mathrm{x}) \\
0, \text { ifj } \neq \mathrm{p}^{\mathrm{k}}(\mathrm{x}) .
\end{array}\right.
\end{aligned}
$$

Then, we have

$$
\sum_{\mathrm{j} \in \mathcal{J}} \eta_{\mathrm{j}}^{\prime}(\mathrm{x}) \frac{1}{\mathrm{C}_{\mathrm{j} j \mathrm{j}}(\mathrm{x}) \phi_{\mathrm{j}}(\mathrm{k})} \geq \sum_{\mathrm{j} \in \mathcal{J}} \eta_{\mathrm{j}}^{\mathrm{k}}(\mathrm{x}) \frac{1}{\mathrm{C}_{\mathrm{j}} \mathrm{r}_{\mathrm{j}}(\mathrm{x}) \phi_{\mathrm{j}}(\mathrm{k})}
$$

Hence, $\left\langle\nabla L\left(\eta^{k}\right), \eta^{s}-\eta^{k}\right\rangle \geq 0$. Therefore, $\eta^{k}$ is an optimal IoT device association in the kth iteration.

As we know, all BSs will estimate and broadcast the traffic load vector $\boldsymbol{\rho}$ and the compuitng load vector $\hat{\boldsymbol{\rho}}$ iteratively, which can be employed by IoT devices to select the suitable BSs. Thus, we need to prove the convergence of $\boldsymbol{\rho}$ and $\hat{\boldsymbol{P}}$ for the proposed scheme.

Theorem 2. At the BS side, the estimated traffic load vector $\mathbf{P}$ and computing load vector $\hat{\mathrm{P}}$ converge to the optimal load vectors $\rho^{*}$ and $\hat{\rho}^{*}$, respectively, such that $\mathrm{L}(\tilde{\mathrm{\eta}})$ is minimized.

Proof: As shown in Lemma $3, \tilde{\eta}^{\mathrm{k}+1}-\tilde{\eta}^{\mathrm{k}}$ provides a decent direction of ${ }^{L}(\tilde{\eta})$ at $\tilde{\eta}^{k}$ and hence $L(\tilde{\eta})$ gradually decreases in each iteration. Since $L(\tilde{\eta})>0, \tilde{\eta}$ will eventually converge when $L(\tilde{\eta})$ is minimized.

According to Eq. (6) and (7), the traffic loads of BSs $P$ and the computing loads of fog nodes $\hat{\rho}$ are determined by $\tilde{\eta}$. Thus, when the intermediate IoT device association $\tilde{\eta}$ converges, the advertised traffic load vector $P$ and computing load vector $\hat{\boldsymbol{\rho}}$ also converge at the same time.

Lemma 2. Based on the optimal advertised traffic load vector $\rho$ and computing load vector $\hat{\rho}$, the IoT device side algorithm yields the optimal IoT device association for the load balancing problem in the feasible set $\mathrm{F}$.

Proof: The proof of this lemma is similar to the proof of Theorem 1.

As LAB is a gradient algorithm, which is a classic algorithm for convex problems, the number of iterations required to ensure convergence can be found in [15].

\section{PERFORMANCE EVALUATION}

The simulation of our algorithm is performed using NS-2 and SUMO simulators. Here considered the following metrics to measure the performance of our new approach:

End-to-End Delay: Time taken for a packet to be transmitted across a network from source to destination.

Collision Ratio: The number of packets colliding across a network before reaching the destination.

Probability of Message Delivery: Probability of the message is delivered to the receiver.

\section{COMPARISON OF OUR HYBRID FOG}

As mentioned above, compared our fog computing approach with PrEPARE and fog-NDN with mobility. The metrics considered for simulations are 1) End-to-end delay, 2) Collision ratio and 3) Probability of message delivery. The probability of message delivery of our fog approach was observed to be higher high due to the location awareness with the help of a base station. Hence, it provides the guaranteed message to the vehicles situated in obstacle shadowing region. Whereas in PrEPARE and Fog-NDN with mobility, a message drop is likely during transmission. In addition, the probability of message delivery is low as the number of users increases, which affects the system load, represented.

Here it is compared the performance of our proposed algorithm in terms of the end-to-end delay, collision ratio and probability of message delivery with CLBP, CMDS, and flooding protocols. The probability of message delivery using other protocols is relatively low when compared to our approach, as represented in Fig. 1. 


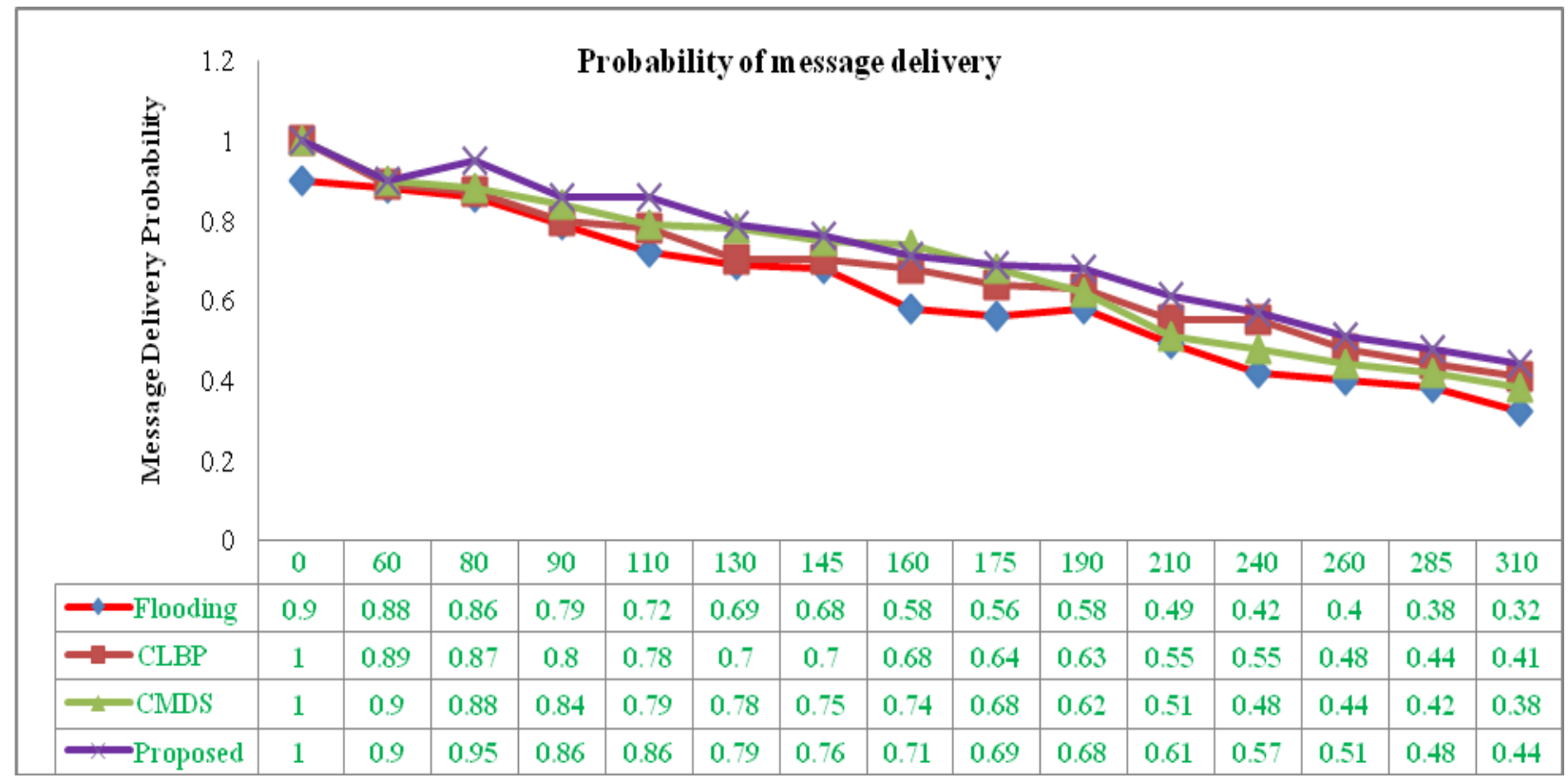

Fig. 1: Probability of message delivery

In CLBP and flooding the messages are disseminated using a multi-hop technique which makes it more likely that a message is dropped in the obstacle shadowed regions. The messages are transmitted using mobile gateways in the CMDS protocol, but mobile gateways are used in transmitting critical messages in between a vehicle and the cloud environment. As a result, this may lead to a message failure situation. In our proposed novel approach, the messages are transmitted to the vehicles with the help of a fog layer in shadowed regions which ensures guaranteed message delivery and thus, it outperforms other protocols by increasing the probability of message delivery.

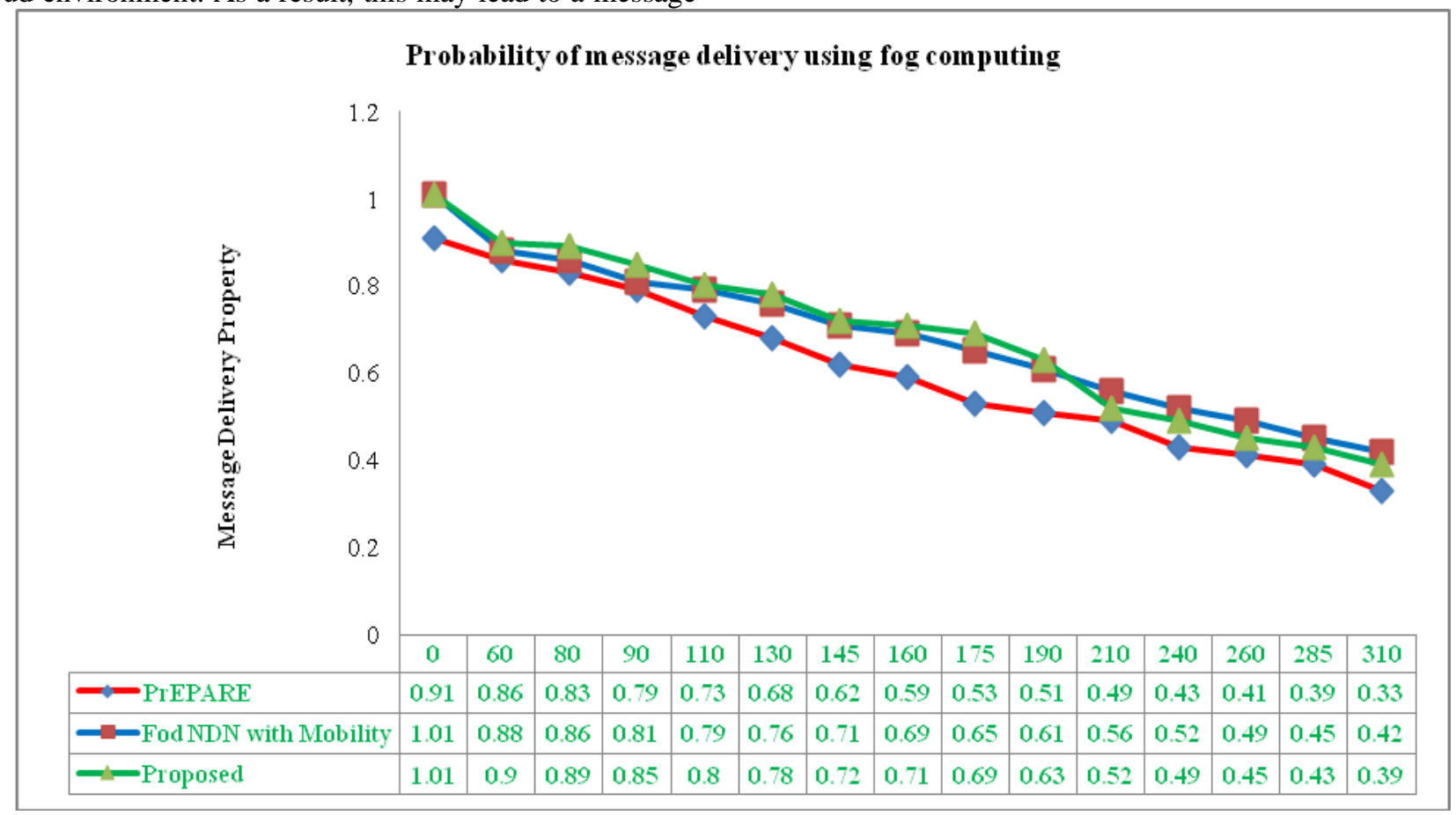

Fig. 2: Probability of message delivery using fog computing

End-to-end delay of PrEPARE and fog-NDN with mobility was observed to be higher due to the various delays associated with message transmission. But in our fog approach, knowledge of nearby vehicles including the position significantly reduces the route setup time and propagation time across a network. Hence, it delivers the message much faster when compared to other protocols. The end-to-end delay increases when the number of users increases in a system due to numerous packets that need to be transmitted at a given time, represented in Fig. 2.

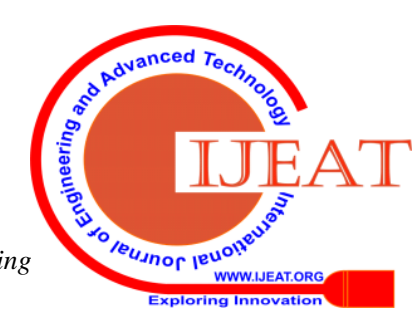




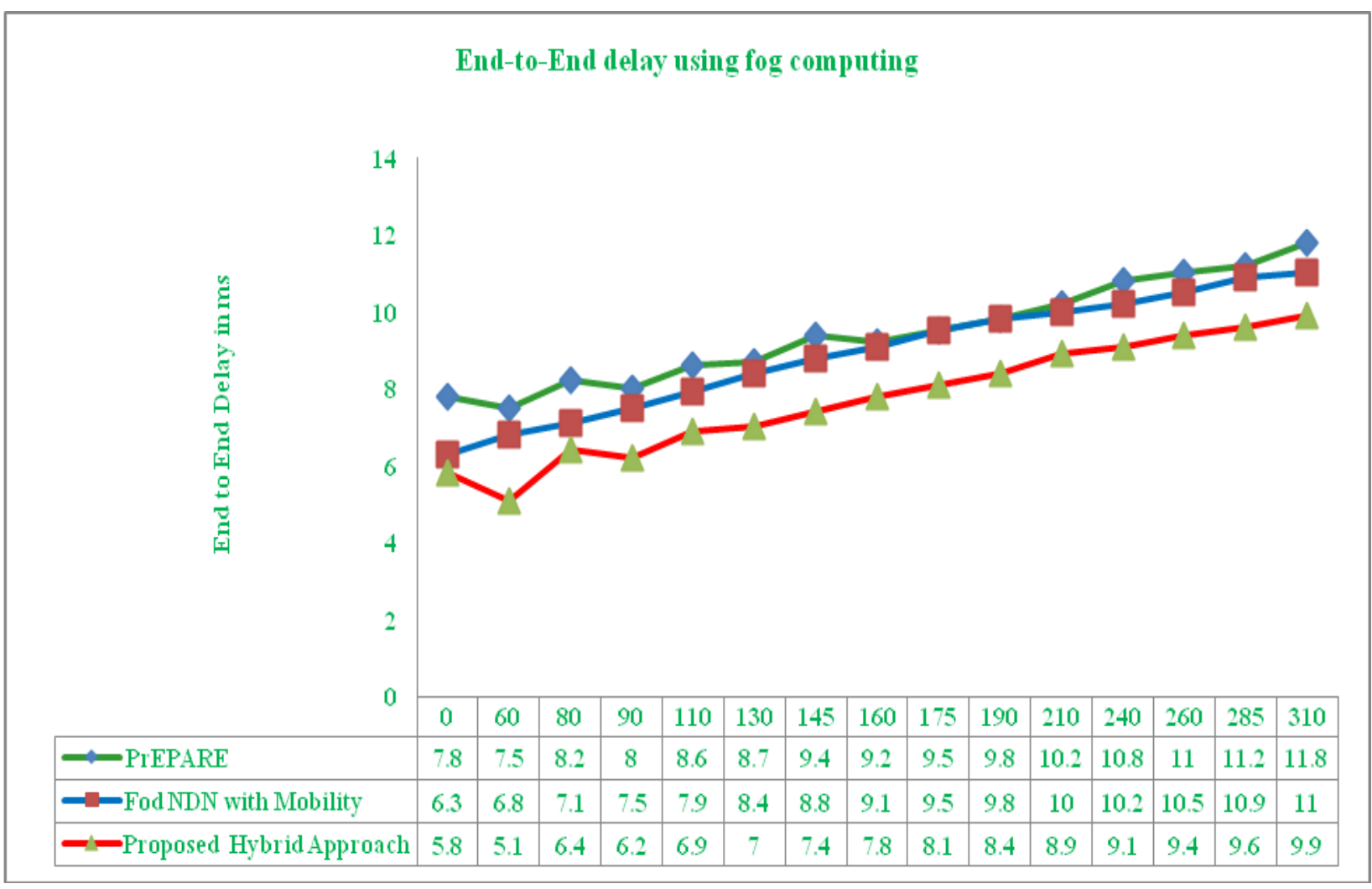

Fig. 3: End-to-End delay using fog computing

The collision ratio of our fog computing approach was observed to be lower due to the number of packets (i.e, critical messages) delivered to the nearby vehicles at a given time. This is because our fog approach disseminates critical messages to the vehicles situated in the obstacle shadowing region. But PrEPARE and fog-NDN with mobility rely upon mobile nodes including fog for transmission of messages which results in a packet collision, represented in Fig. 4.

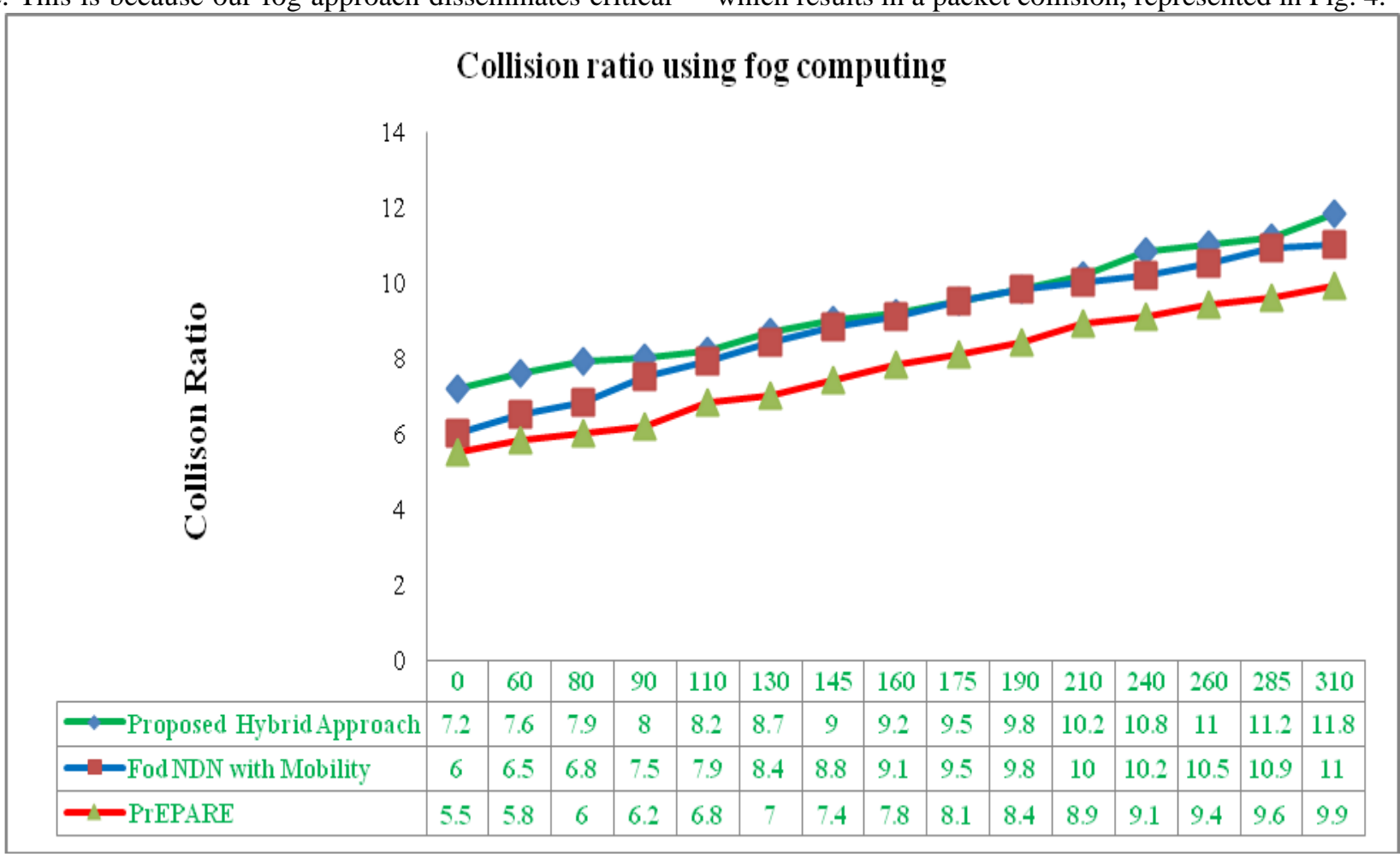

Fig. 4: Collision ratio using fog computing

\subsection{COMPARISON OF OUR HYBRID FOG APPROACH WITH OTHER PROTOCOLS}




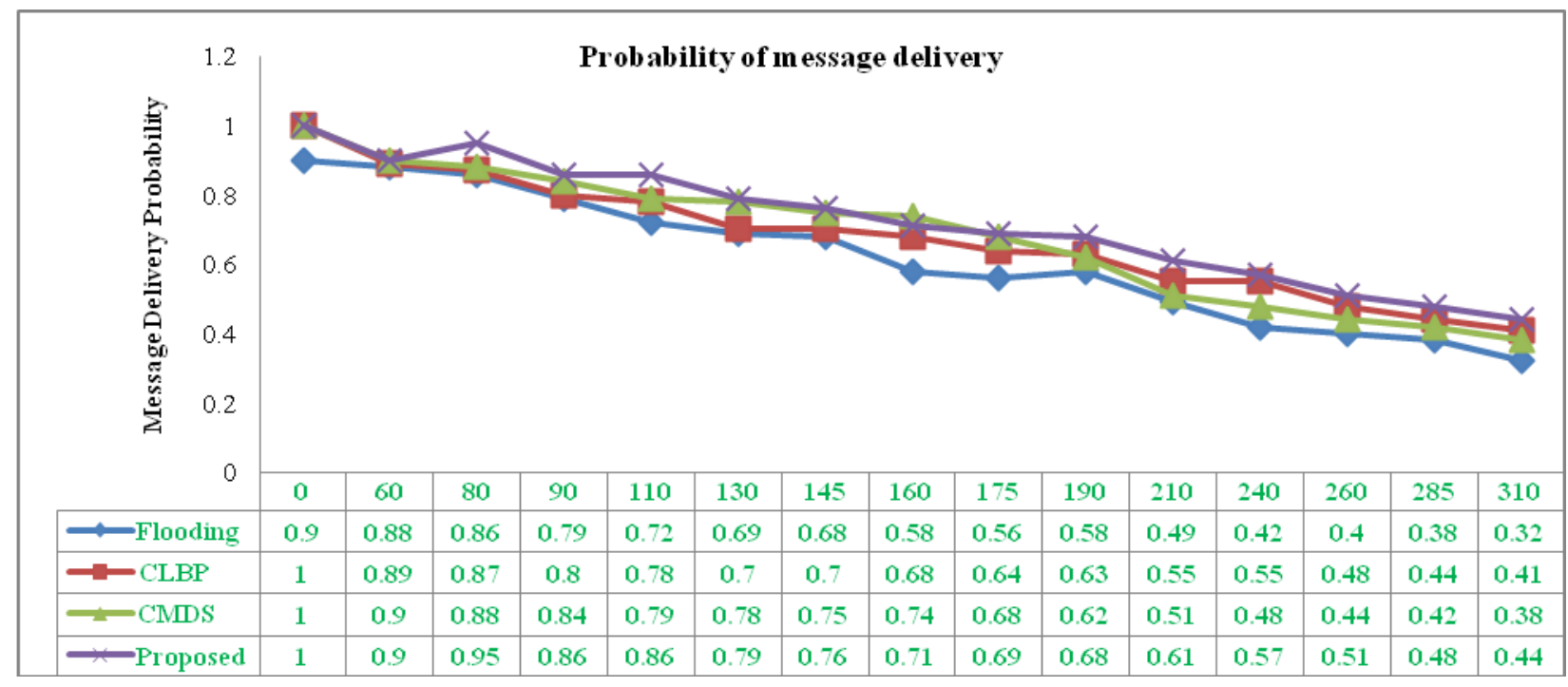

Fig. 5: Probability of message delivery

Here it is compared the performance of our algorithm in terms of the end-to-end delay, collision ratio and probability of message delivery with CLBP, CMDS, and flooding protocols. The probability of message delivery using other protocols is relatively low when compared to our proposed approach, as represented in Fig. 5. In CLBP and flooding the messages are disseminated using a multi-hop technique which makes it more likely that a message is dropped in the obstacle shadowed regions. The messages are transmitted using mobile gateways in the CMDS protocol, but mobile gateways are used in transmitting critical messages between a vehicle and the cloud. As a result, this may lead to a message failure situation. In our approach, the messages are transmitted to the vehicles with the help of a fog layer in shadowed regions which ensures guaranteed message delivery and thus, it outperforms other protocols by increasing the probability of message delivery.

A comparison of the end-to-end delay of proposed approach with other schemes is presented in Fig. 6. The results showed that the end-to-end delay of our approach is lower than that of the CLBP, CMDS, and flooding algorithms. In our proposed approach, messages are disseminated to other vehicles with the help of a base stations and RSUs in the fog layer. The base station is aware of the location of all vehicles situated in its transmission range which helps in reducing the time taken for an initial setup across a network from source to destination and thus, the end-to-end delay of the Hybrid-Vehfog is relatively lower than other protocols.

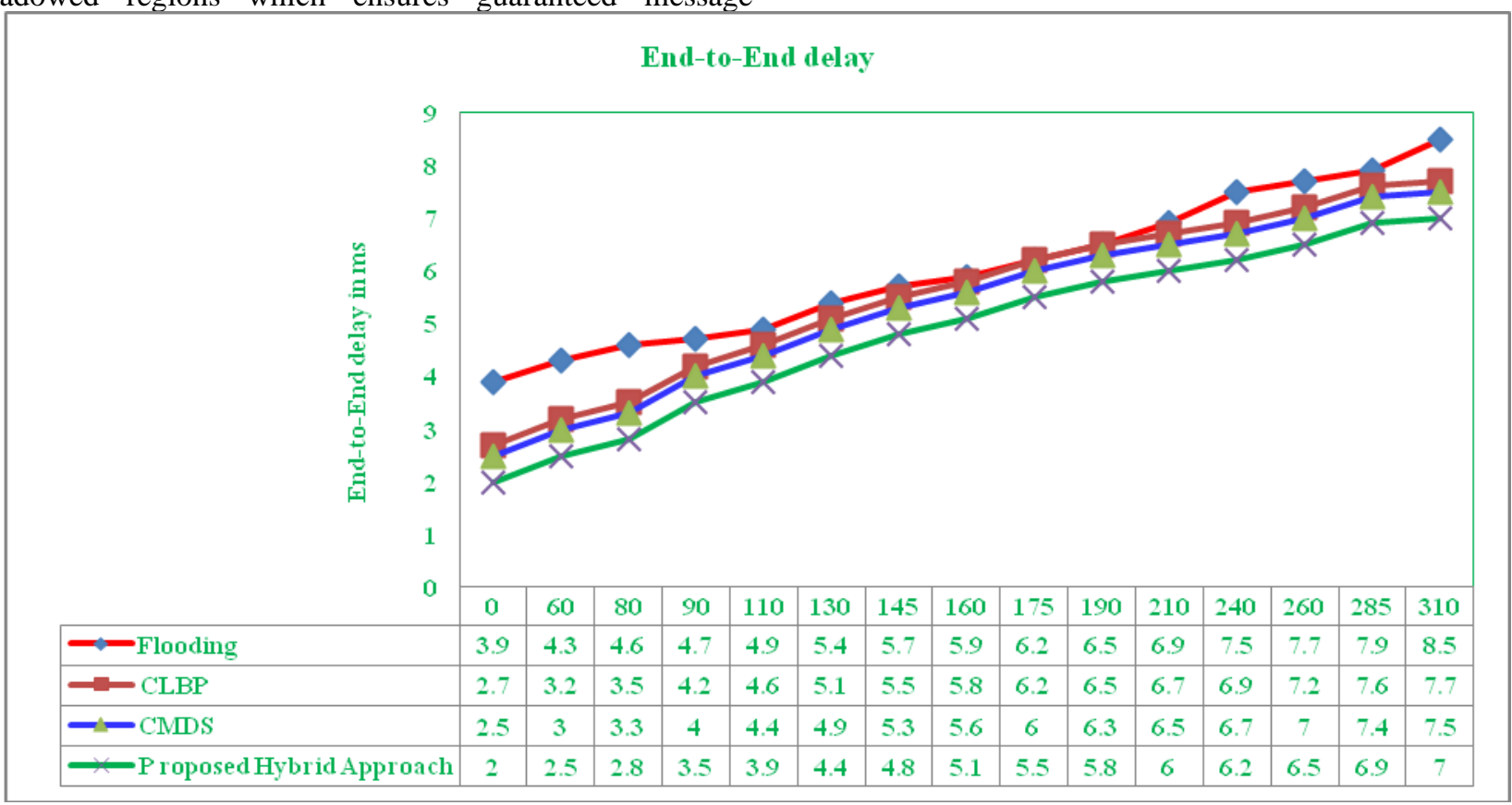

Fig.6: End-to-End delay

In order to observe the number of packets that were dropped without reaching their destination, we broadcasted the critical messages to nearby vehicles at a time interval (t1). 


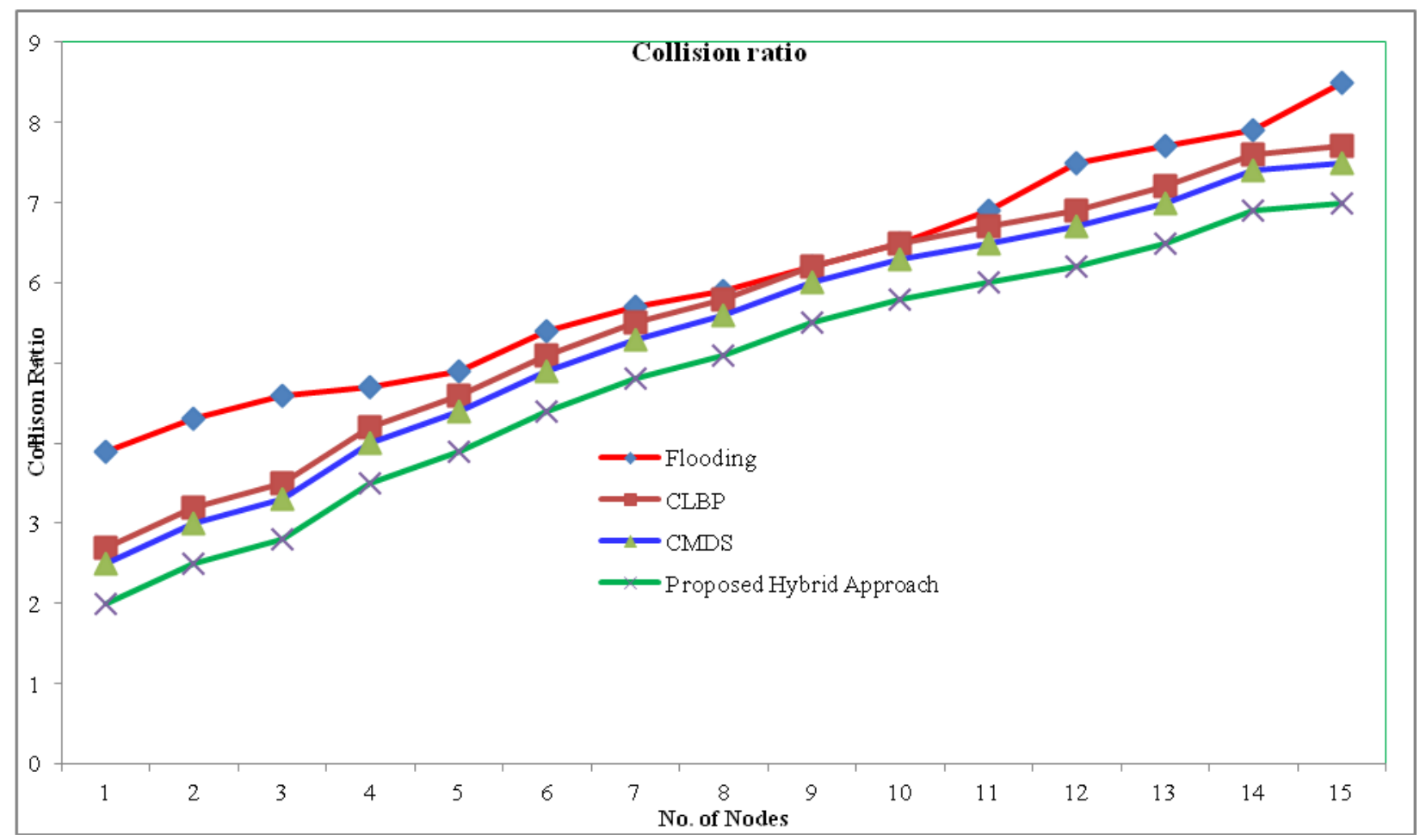

Fig. 7: Collision ratio

The collision ratio of our approach was observed to be lower than that of the CLBP, CMDS, and flooding protocols. Our approach provides guaranteed message delivery to the targeted vehicles whereas in other schemes there is a high chance of message transmission failure, a situation which leads to the retransmission of input messages. Accordingly, the number of packets generated in a time interval (t1) increases, which in turn increases the collision ratio, as represented in Fig. 7.

\section{CONCLUSION}

Our hybrid algorithm dynamically adapts to changes in an environment and benefits in efficiency with robust drone vehicle deployment capability as needed. Performance of our routing protocol is carried out in Network Simulator (NS-2) and Simulation of Urban Mobility (SUMO) simulators. The results showed that Hybrid fog routing outperformed Cloud-assisted Message Downlink Dissemination Scheme (CMDS), Cross-Layer Broadcast Protocol (CLBP), PEer-to-Peer protocol for Allocated REsource (PrEPARE), Fog-Named Data Networking (NDN) with mobility, and flooding schemes at all vehicle densities and simulation times.

\section{REFERENCE}

1. F. Bonomi, et al.(2012), "Fog computing and its role in the internet of things," in Proceedings of the First Edition of the MCC Workshop on Mobile Cloud Computing, ser. MCC '12. New York, NY, USA: ACM, pp. 13-16.

2. Cisco, "Cisco delivers vision of fog computing to accelerate value from billions of connected devices," Cisco Press Release, Jan 2014.

3. T. H. Luan, et al.(2015), "Fog Computing: Focusing on Mobile Users at the Edge," ArXiv e-prints, Feb. 2015.

4. C. Stojmenovic et al(2014), "The fog computing paradigm: Scenarios and security issues," in Computer Science and Information Systems (FedCSIS), 2014 Federated Conference on, Sept 2014, pp. 1-8.

5. R. Deng, et al.(2016), "Optimal workload allocation in fog-cloud computing towards balanced delay and power consumption," IEEE Internet of Things Journal, May 2016.
6. A. Targhetta, et al.(2014), "The design space of ultra-lowenergy asymmetric cryptography," in Performance Analysis of Systems and Software (ISPASS), 2014 IEEE International Symposium, pp. 55-65.

7. R. Lu,(2010), "Pi: A practical incentive protocol for delay toleran networks," Wireless Communications, IEEE Transactions on, vol. 9, no. 4, pp. 1483-1493.

8. A. Pentland, et al.(2004), "DakNet: rethinking connectivity in developing nations," Computer, vol. 37, no. 1, pp. 78-83.

9. L. Gao, et al.(2015), "Delay Tolerant Networks and Their Applications". Springer Publishing Company, Incorporated, 2015.

10. Y. Cao et al.(2013), "Routing in delay/disruption tolerant networks: A taxonomy, survey and challenges," Communications Surveys Tutorials, IEEE, vol. 15, no. 2, pp. 654-677.

11. A. Pentland, et al(2004), "DakNet: rethinking connectivity in developing nations," Computer, vol. 37, no. 1, pp. 78-83.

12. L. Gao, et al.(2013), "Multidimensional routing protocol in human-associated delay-tolerant networks," Mobile Computing, IEEE Transactions on, vol. 12, no. 11, pp. 2132-2144.

13. A. Targhetta, et al.(2014), "The design space of ultra-low energy asymmetric cryptography," in Performance Analysis of Systems and Software (ISPASS), 2014 IEEE International Symposium on, pp. 55-65.

14. X. Zhuo, et al.(2014), "An incentive framework for cellular traffic offloading," Mobile Computing, IEEE Transactions on, vol. 13, no. 3, pp. 541- 555 .

15. S. Parameswari, et al.(2020), "Fog Route: Distribution of Data using Delay Tolerant Network", Journal of Engineering and Applied Sciences 15 (2): 508-515, 2020,ISSN: 1816-949X. 